\title{
DISTRIBUTION OF CRACK-TIP STRESSES DURING FATIGUE LOADING WITH AN OVERLOAD EVENT: ROLE OF INITIAL CRACK-TIP SHAPE, PLASTIC COMPRESSIBILITY AND MATERIAL SOFTENING
}

\author{
Satyabrat Pandey, Debashis Khan, Intaf Alam \\ Indian Institute of Technology (BHU) Varanasi, Department of Mechanical Engineering, Varanasi, India \\ e-mail:dkhan.mec@itbhu.ac.in
}

\begin{abstract}
This paper deals with the influence of initial crack-tip shape, plastic compressibility and material or strain softening on near-tip stress-strain fields for mode I crack when subjected to fatigue loading with an overload event under plane strain and small scale yielding conditions. A finite strain elastic-viscoplastic constitutive equation with a hardening-softening-hardening hardness function is taken up for simulation. For comparison, a bilinear hardening hardness function is also considered. It has been observed that the near-tip crack opening stress $\sigma_{y y}$, crack growth stress $\sigma_{x x}$, and hydrostatic stresses are noticeably controlled by the initial crack tip shape, plastic compressibility, material softening as well as the overload event. The distribution pattern of different stresses for a plastically compressible hardening-softening-hardening solid appears to be very unusual and advantageous as compared to those of traditional materials. Therefore, the present numerical results may guide material scientists/engineers to understand the near-tip stress-strain fields and growth of a crack in a better way for plastically compressible solids, and thus may help to develop new materials with improved properties.
\end{abstract}

Keywords: fatigue loading, finite deformation, mode I crack, stress-strain field, plastically compressible solid, material softening

\section{Introduction}

Service life assessment of engineering components and structures with cracks or similar defects has been a target of material scientists/engineers since long. In practice, for such components and structures, variable amplitude fatigue is more common in comparison to constant amplitude fatigue loading or monotonic loading. Keeping this in mind, a plenty of research works, over the years, have been undertaken to characterize crack growth behavior following an overload or underload in fatigue loading. The present state of knowledge in this context may be summarized as (Sadananda et al., 1999; Steuwer et al., 2010): (a) overload causes retardation whereas underload causes acceleration in the fatigue crack growth rate, (b) after an overload, there is a sudden acceleration phase in the crack growth before prolonged retardation, (c) the amount of retardation is found to depend on the overload ratio, number of overloads, load ratio and a baseline value of the stress intensity factor range, (d) after an overload or underload event in a load spectrum, a certain number of cycles should be applied for reestablishing the original steady state crack growth rate. These outcomes have been demonstrated in terms of residual stress, crack-tip blunting, crack closure, work hardening, crack branching and reverse yielding, etc. However, in spite of the considerable amount of investigations focused on the effects of overload and underload, the mechanisms of overload/underload fatigue crack growth are not completely understood and the issues are still the subject of debate.

While considerable amounts of literature on fatigue crack growth studies are available for materials like steels, aluminum alloys, titanium alloys, etc., there are few studies on relatively 
new materials like metallic foams, toughened structural polymers, transformation toughened ceramics, etc. in the open literature (Mohan et al., 2013). Although, in classical plasticity theory, the role of hydrostatic pressure is typically neglected, nevertheless, experiments on these new materials demonstrate pressure-sensitive yielding and plastic dilatancy. As for these materials, there is a volume change during deviatoric loading, they are also known as plastically compressible materials. These new materials have great potential application areas and, till now, only a little has been explored; like, in energy absorption mechanism, impact resistance, thermal and electrical interfaces, various bioimplants, etc. Albeit, it is identified that the phenomena of plastic compressibility and strain softening can lead to noteworthy variation in the stress-strain fields and crack-tip deformation, the studies, for example, by Hutchens et al. (2011), Needleman et al. (2012), Mohan et al. (2013), Khan et al. (2017), Singh and Khan (2018), Alam et al. (2019) have provided some basic understanding under monotonic loading, and only a little under constant amplitude fatigue loading on the topic. Thus there is still room for investigations of such materials particularly with respect to fatigue loading applications. Furthermore, Rozumek et al. (2006) studied the influence of crack-tip curvature on fatigue crack growth experimentally for steel and aluminum, and they observed higher fatigue crack growth for a blunter notch. In the study of Alam et al. (2019), similar observations were also revealed. To find out the root cause of this bizarre observation, complete evolution of the near-tip stress-strain field is necessary, however, in the works of Rozumek et al. (2006) and Alam et al. (2019), the same was not discussed in detail. The crack-tip deformation and stress field studies are not only fundamental for understanding of the crack propagation but also for satisfactory development of predictive tools. Till now, no numerical simulation has successfully explained or reproduced the crack-tip deformation and fields during fatigue loading for various crack-tip profiles except for a circular crack-tip. In the presence of cyclic or fatigue loading with an overload event, the influence of initial crack-tip shape variation on the degree of near-tip fields is an unexplored and exciting area to be looked into. Furthermore, also unknown is the possible outcome in the non-circular crack-tip deformation and fields of plastically compressible solids when fatigue loadings are in force. In addition, if there is strain softening during the deformation, it is likely that the study may make available some new interesting findings. It is therefore of great practical importance to reveal the near-tip fields owing to the combined effect of initial crack-tip shape, plastic compressibility and strain hardening/softening when the fatigue loadings with the overload are in force. The findings of the present work may also encourage researchers working in this area to design new materials with improved properties.

In this study, we perform plane strain finite element finite deformation analyses of mode I crack under fatigue loading with a constant as well as variable amplitude (with overload) for a variety of practical crack-tip shapes. The present computations are limited to plastic normality flow rule and small scale yielding conditions. We particularly investigate here the impact of initial crack-tip shape, plastic compressibility, material softening and fatigue loading with an overload on near-tip fields in a hardening-softening-hardening material which may represent foams, vertically aligned carbon nanotubes (VACNTs), etc. For comparison, some results related to a bilinear hardening material which may represent many metals have also been considered.

\section{Modelling issues}

The constitutive model used here is the same as that given in (Mohan et al., 2013; Khan et al., 2017; Singh and Khan, 2018; Alam et al., 2019). Here, the rate of deformation tensor $\mathbf{d}$ is written as the sum of the isotropic elastic part $\mathbf{d}^{e}=\mathbf{L}^{-1}: \widetilde{\boldsymbol{\tau}}$ characterized by Young's modulus $E$ and Poisson's ratio $\nu$ and the viscoplastic plastic part $\mathbf{d}^{p}$. Here, the $\widetilde{\boldsymbol{\tau}}$ represents the Jaumann rate 
of Kirchhoff's stress. The elastic strains are assumed to be very small, and the plastic part $\mathbf{d}^{p}$ is given as

$$
\mathbf{d}^{p}=\frac{3}{2} \frac{\ddot{\varepsilon}_{p}}{\sigma_{e}} \mathbf{p}
$$

where

$$
\mathbf{p}=\boldsymbol{\tau}-\alpha \operatorname{tr}(\boldsymbol{\tau}) \mathbf{I} \quad \dot{\varepsilon}_{p}=\dot{\varepsilon}_{0} \sqrt[m]{\frac{\sigma_{e}}{g}}
$$

where $\alpha$ denotes the parameter related to plastic compressibility, $\dot{\varepsilon}_{0}$ and $m$ correspond, respectively, to the reference strain rate and rate hardening exponent, and the hardness function $g\left(\varepsilon_{P}\right)$ is given as

$$
g\left(\varepsilon_{p}\right)=\sigma_{0} \begin{cases}1+h_{1} \varepsilon_{p} & \varepsilon_{p}<\varepsilon_{1} \\ 1+h_{1} \varepsilon_{1}+h_{2}\left(\varepsilon_{p}-\varepsilon_{1}\right) & \varepsilon_{1}<\varepsilon_{p}<\varepsilon_{2} \\ 1+h_{1} \varepsilon_{1}+h_{2}\left(\varepsilon_{2}-\varepsilon_{1}\right)+h_{3}\left(\varepsilon_{p}-\varepsilon_{2}\right) & \varepsilon_{p}>\varepsilon_{2}\end{cases}
$$

where $\sigma_{0}$ represents the reference stress and $\sigma_{e}$ the effective stress given by

$$
\sigma_{e}^{2}=\frac{3}{2} \boldsymbol{\tau}: \mathbf{p}=\frac{3}{2}\left[\boldsymbol{\tau}: \boldsymbol{\tau}-\alpha(\operatorname{tr}(\boldsymbol{\tau}))^{2}\right]
$$

The term within the square brackets of above equation (2.3) is non-negative when $\alpha \leqslant 1 / 3$. For $\alpha=1 / 3$, the constitutive equation corresponds to that of a plastically incompressible von Mises solid.

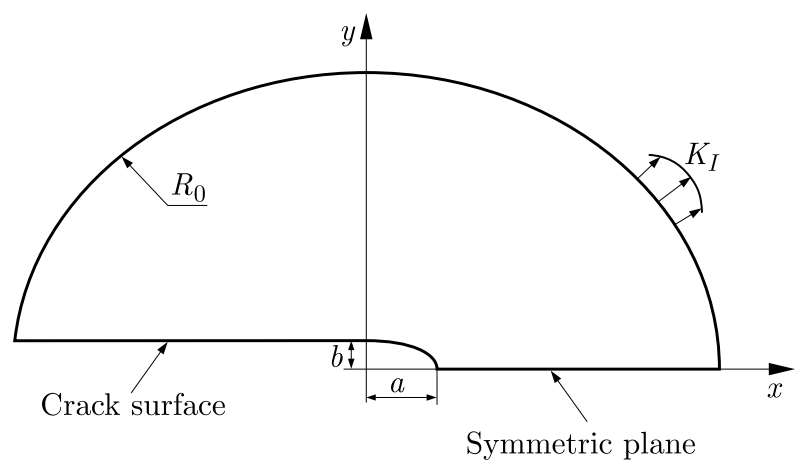

Fig. 1. Geometry and the elliptical crack-tip used for the simulation

The numerical analysis is performed for a semi-circular geometry, Fig. 1 . The outer radius $R_{0}$ of the geometry is 2.0 in any arbitrary units. For describing various crack-tip profiles, we consider a similar kind of an elliptical arc as taken in our earlier work (Alam et al., 2019). This sort of the crack profile is more realistic as compared to a crack with a circular arc tip usually adopted for numerical simulation or a mathematically sharp crack assumed for academic interest. In Fig. 1, $a$ is the semi-major axis and $b$ is the semi-minor axis of the ellipse, and they are in the same arbitrary units as those of $R_{0}$. The ratio $a / b$ is employed for generating several crack-tip shapes. Traction-free crack surfaces are assumed for the analysis, and on the line $y=0$, symmetry boundary conditions are enforced. As used in our earlier works, for example (Singh and Khan, 2018) for displaying the localization, the finite element grid is comprised of rectangular elements and each rectangular element is further consisting of four-crossed linear displacement triangular elements. Finite elements employed here are shown in Fig. 2 (only the quadrilaterals are shown). 
(a)

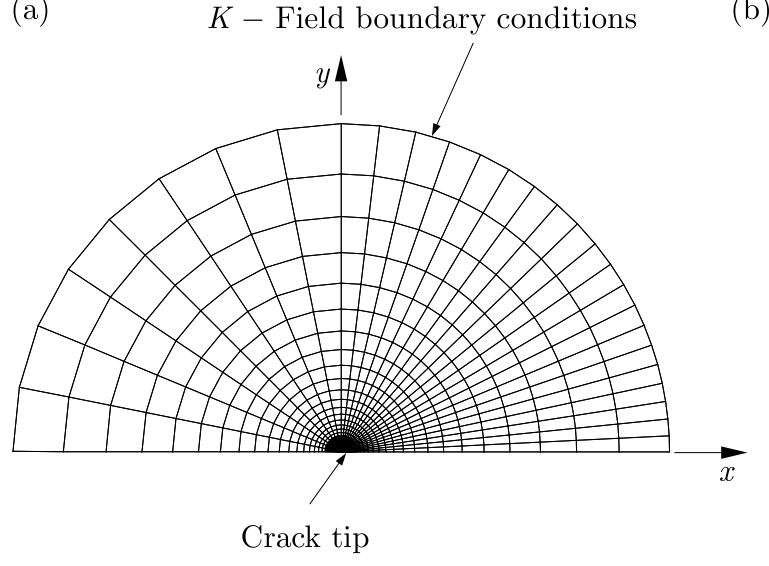

(b)

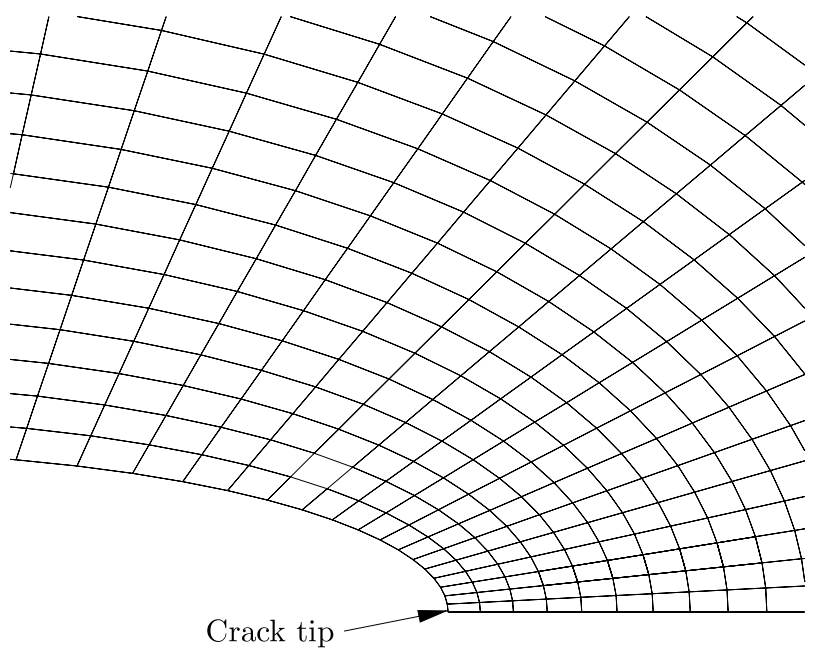

Fig. 2. Typical finite element mesh for the simulation: (a) full mesh, (b) near-tip mesh

Materials B and E (from Mohan et al., 2013) have been chosen here for computational simulation, Fig. 3. Material B, being a bilinear hardening solid may represent several metals, whereas material E, a trilinear hardening-softening-hardening material may represent foams and VACNTs, (Hutchens et al., 2011). The parameters, $h_{1}=24$ and $\varepsilon_{1}=0.085$ are used for both the materials. For material $\mathrm{B}, h_{2}=h_{3}=5.0$ and $\varepsilon_{2}$ value is irrelevant now whereas for material $\mathrm{E}$, $h_{2}=-3.90, h_{3}=15.0$ and $\varepsilon_{2}=0.6$. The constant material parameters used in the simulation are $E / \sigma_{0}=100$, Poisson's ratio $\nu=0.25$, reference strain rate $\dot{\varepsilon}_{0}=1$ and the rate hardening exponent $m=0.02$.

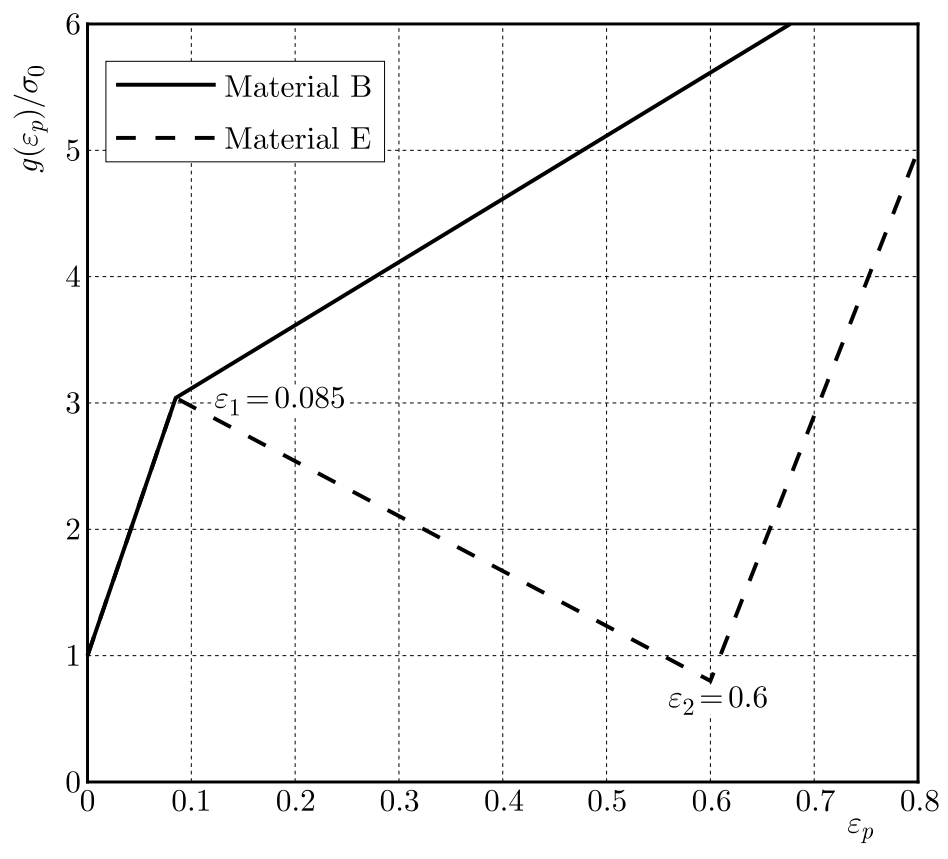

Fig. 3. The hardness functions for materials $\mathrm{B}$ and $\mathrm{E}$

The finite element finite deformation formulation is the same as we used in some of our earlier works, for example, in Singh and Khan (2018), and therefore we are not repeating it 
here. On the outer boundary of the semi-circular geometry, isotropic and linear elastic mode I displacement field is prescribed using equations (2.4). Plane strain conditions are assumed

$$
\begin{aligned}
& \dot{u}_{1}=\frac{2(1+\nu) \dot{K}_{I}}{E} \sqrt{\frac{R}{2 \pi}} \cos \frac{\theta}{2}\left(1-2 \nu+\sin ^{2} \frac{\theta}{2}\right) \\
& \dot{u}_{2}=\frac{2(1+\nu) \dot{K}_{I}}{E} \sqrt{\frac{R}{2 \pi}} \sin \frac{\theta}{2}\left(2-2 \nu-\cos ^{2} \frac{\theta}{2}\right)
\end{aligned}
$$

where $R=\sqrt{x^{2}+y^{2}}, \theta=\tan ^{-1}(y / x)$ and $K_{I}$ is the stress intensity factor; a dot over it represents the time derivative. The corresponding value of the applied $J$ integral, i.e. $J_{a p p}$, is provided for a small scale yielding by Rice (1968) as $J_{a p p}=K_{I}^{2}\left(1-\nu^{2}\right) / E$.

In the present investigation, the maximum load $\left(K_{I}\right)_{\max }=1.5$ was applied on the outer boundary of the geometry. Total ten load cycles were applied. Here, $\left(K_{I}\right)_{\min }=0$ and $\left(K_{I}\right)_{\max }=1.5$ were used in a form of a triangle wave during one cycle. An overload of $\left(K_{I}\right)_{\text {overload }}=1.5\left(K_{I}\right)_{\max }$ was applied to the 5 th cycle. A constant value $\dot{K}_{I} / \sigma_{0} \dot{\varepsilon}_{0} \sqrt{b_{0}}=31.62$ was prescribed with $\dot{K}_{I}=1 \mathrm{MPa} \sqrt{\mathrm{m}} / \mathrm{s}$. In order to update the constitutive equation, we used here the rate tangent modulus method of Peirce et al. (1984), and also a time step size of $d t=0.0002 \mathrm{~s}$ was used in the simulation.

\section{Numerical results and discussion}

\subsection{Mesh convergence and comparison with earlier results}

With the present visco-plasticity material model as mentioned in Section 2, mesh convergence study of the semi-circular geometry was carried out without any special crack-tip element. Calculations were performed with mesh densities consisting of $24 \times 53,24 \times 63$, and $24 \times 73$ crossed rectangular elements. Near-tip cumulative plastic strain contours were compared. It was revealed that the plastic strain results from $24 \times 63$ and $24 \times 73$ were almost matching as compared to $24 \times 53$. For other mesh densities, also plastic strain solutions were generated. Apart from the initial coarse mesh density, the required solutions were quite steady representing too small differences at higher mesh densities. Comparing the solution accuracy and computation time, the final mesh density was fixed at $24 \times 63$ rectangular elements with 1598 nodes. Sufficiently fine mesh was generated near to the crack-tip. The radial length of a finite element just next to the crack-tip was roughly $b / 10$.

The degree of accuracy level of the present numerical results can be corroborated by noting the similarity of the near-tip stress distributions for a propagating crack of Liu and Drugan (1993). Liu and Drugan (1993) used a monotonic loading and a linear elastic-perfectly plastic material model with Young's modulus $200 \mathrm{GPa}$, Poisson's ratio 0.5 as well as yield stress of $1.173 \mathrm{GPa}$. Using identical conditions, the finite element simulation was run with the present code and, subsequently, the normal and shear stresses were plotted in Fig. 4 . The maximum $\sigma_{x x}$ and $\sigma_{y y}$ values (approximately 1.6 and 2.6, respectively) are almost matching with those of Liu and Drugan (1993). The distribution pattern of all the stress quantities is also almost similar. The minute discrepancy may be owing to dissimilar finite elements used for meshing. In the current work, rectangular elements, each of which is built up of four crossed triangles, have been used, whereas on the other hand for meshing the geometry, conventional quadrilateral elements have been used by Liu and Drugan (1993). The results obtained in Fig. 4 may be considered sufficient to continue additional investigations. 


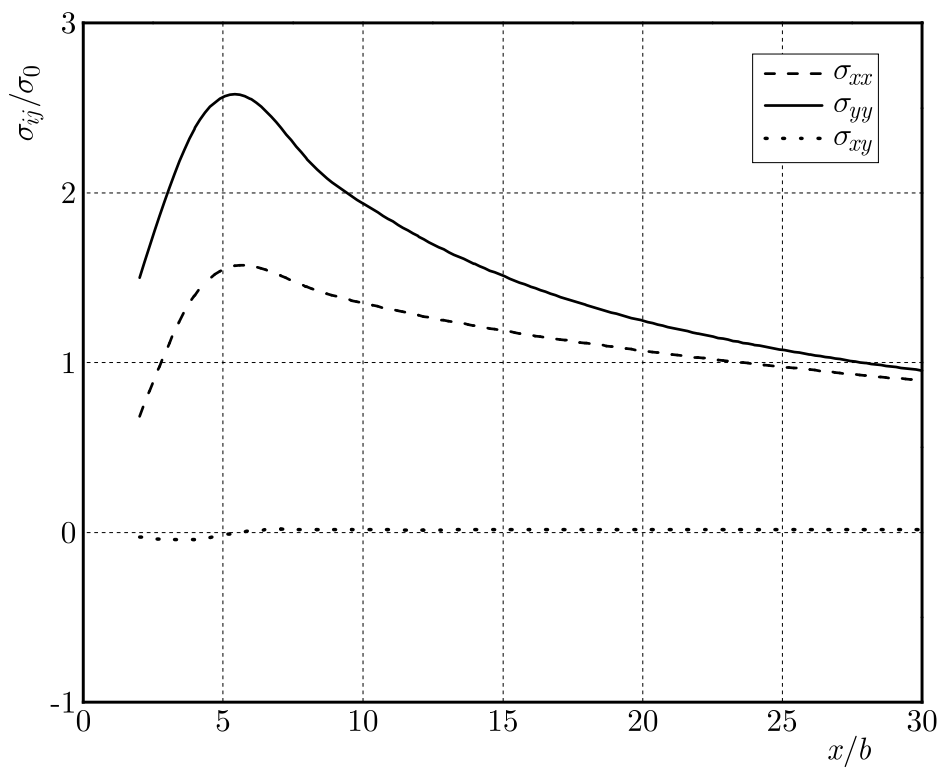

Fig. 4. Near-tip stress distribution for a growing crack (with $\Delta a=0.0086\left(K_{I} / \sigma_{0}\right)^{2}$ ) in a linear elastic and perfectly plastic material

\subsection{Plastic zone shape and size}

Because of the absence of a sharp yield point for the present elastic-viscoplastic constitutive relation, a well defined plastic zone cannot be there, and thus the plastic strain $\varepsilon_{p}$ contour values of 0.001 or 0.002 may be considered to represent the plastic zone size as well as shape. It has already been observed in one of our earlier works (Khan et al., 2017) that the extent of significant plastic strain for bilinear hardening is nearly similar to that of hardening-softeninghardening solids. It has also been studied in this work that the plastic zone shape and size for all the three crack-tip shapes considered (with $a / b=1, a / b=4$ and $a / b=6$ ) are almost same. Therefore, the distributions of plastic strain $\varepsilon_{p}$ corresponding to $K \rightarrow K_{\max }$ and $K \rightarrow K_{\min }$ of the 7th load cycle with and without an overload for material $E$ are only presented here for the crack-tip shape with $a / b=1$, i.e. the circular arc crack, Figs. 5-7. Significant differences in the plastic zone shapes and sizes of plastically incompressible solid and a compressible solid with $\alpha=0.28$ are visible. For the solid with $\alpha=0.28$, the contour of larger strain region $\left(\varepsilon_{p}>0.01\right)$ is enlarged, and the shape of the contour is altered from the kidney-like shape for the plastically incompressible solid, Fig. 5. It is also reflected that the plastic zone is very much sensitive to the overload; the overload makes the plastic zone significantly bigger. Because of the overload, the degree of plastic strain varies in both horizontal and vertical directions, and the contours extend by about a factor of 2.5 along the horizontal axis and 2 along the vertical axis than those for the corresponding constant amplitude loading, Fig. 6. From the outermost radius of the geometry and plastic zone size, the small scale yielding conditions have also been checked and found to prevail here. It is also important to note that the size and shape of the reverse plastic zone in this case are almost the same as compared to those of the forward plastic zone, Fig. 7. Even though the degree of severe plastic strain is not much sensitive to whether the material is being loaded or unloaded, nevertheless, the near-tip stress fields, as will be shown subsequently, are very much sensitive to this.

\subsection{Distribution of crack opening stress $\left(\sigma_{y y}\right)$ along the symmetry plane}

Figure 8 illustrates the near-tip distribution of the crack opening stress $\sigma_{y y}$ along the symmetry plane at low and high peak loads (i.e. $K \rightarrow K_{\max }$ and $K \rightarrow K_{\min }$ ) during the 7 th load 
(a)

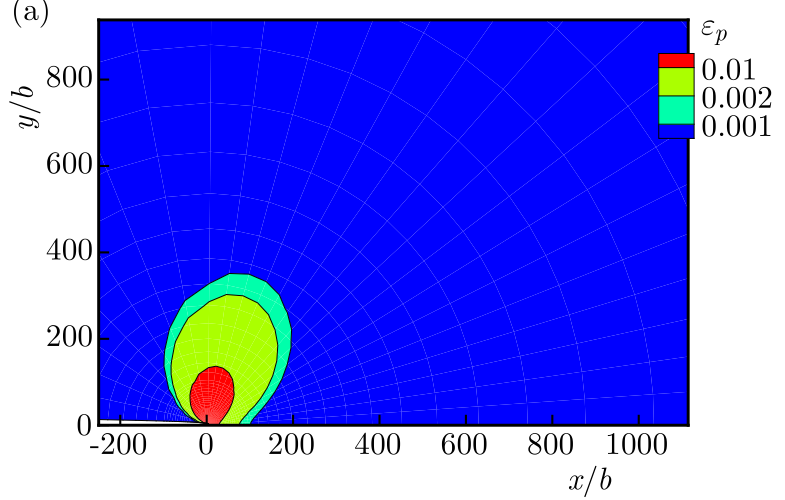

(b)

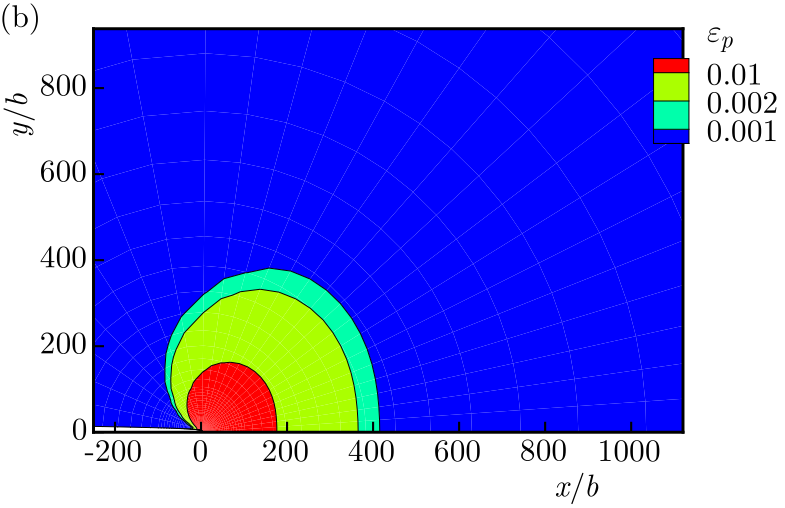

Fig. 5. Forward plastic zones for material $\mathrm{E}$ with $a / b=1, K \rightarrow K_{\max }$ of the 7 th cycle, without overload (a) plastically incompressible, (b) plastically compressible $(\alpha=0.28)$
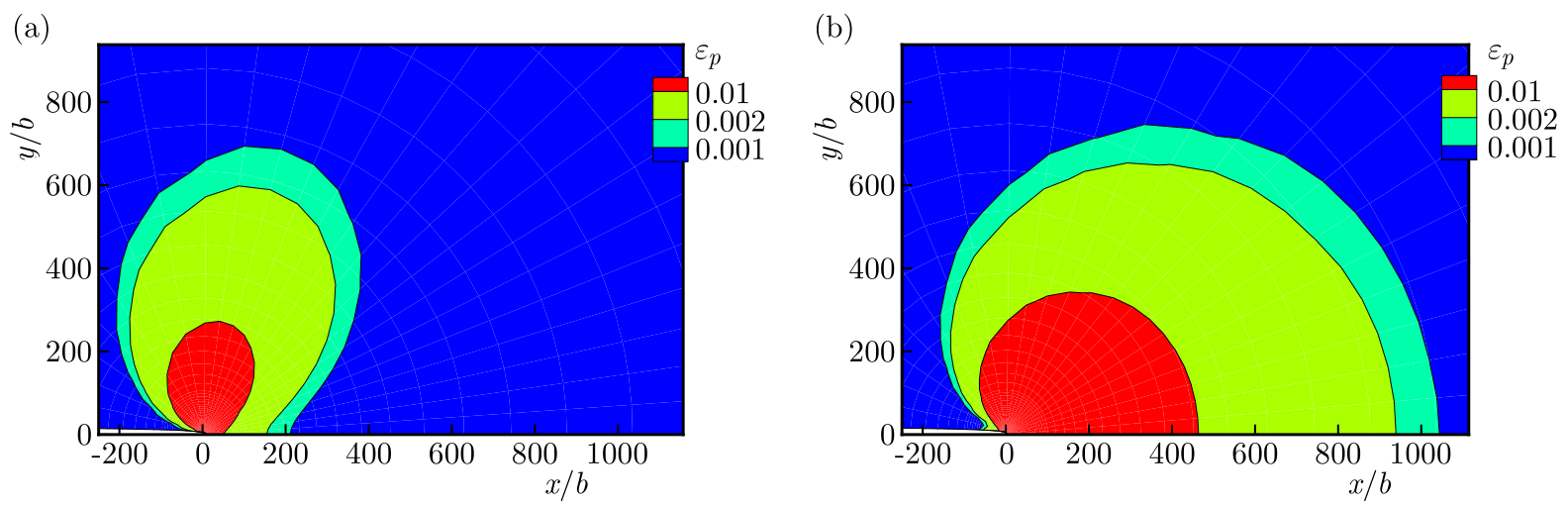

Fig. 6. Forward plastic zones for material E with $a / b=1, K \rightarrow K_{\max }$ of the 7 th cycle, with overload (a) plastically incompressible, (b) plastically compressible $(\alpha=0.28)$

(a)

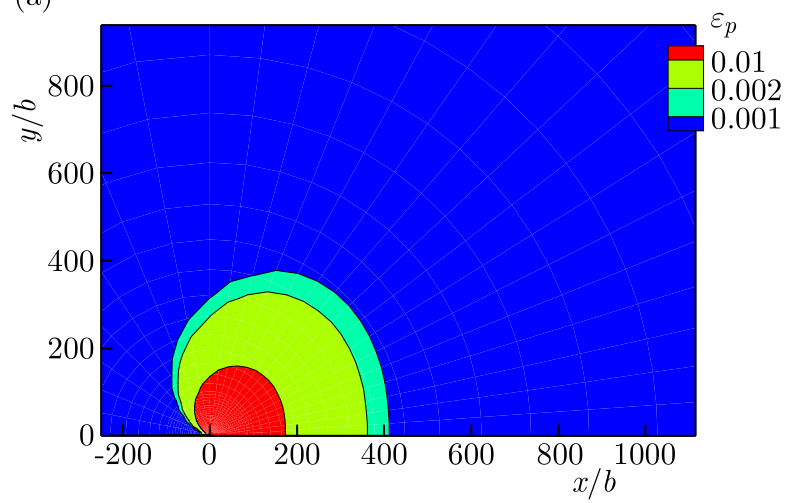

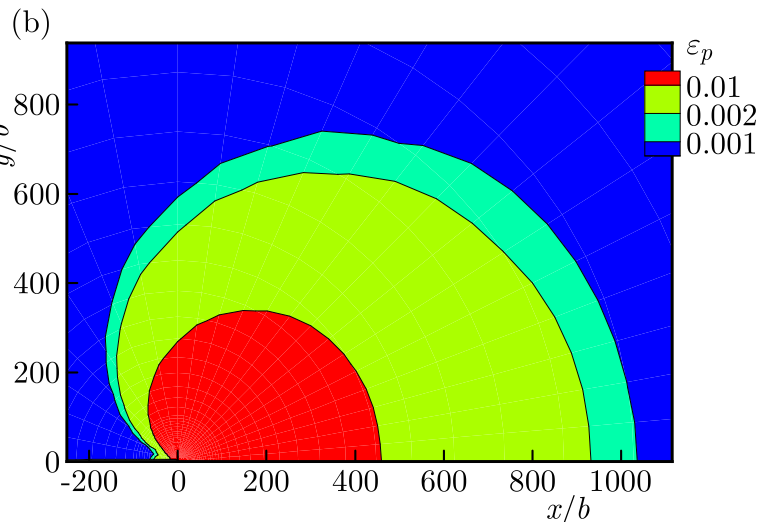

Fig. 7. Reverse plastic zones for material E with $a / b=1$, plastically compressible solid $(\alpha=0.28)$, $K \rightarrow K_{\min }$ of the 7 th cycle (a) without overload, (b) with overload

cycle for material B. For material B, as the plots are nearly similar for both the 1st and 7th cycles, the results are shown for the 7th cycle only. There are differences in the distribution of $\sigma_{y y}$ values for plastically incompressible and compressible solids, and there are also small differences in the distribution when the radius of crack-tip curvature is changed. The crack-tip shape has very little influence on the stress field away from the tip. The near-tip $\sigma_{y y}$ distributions are more or less self-similar in nature, and they match with the results of others for the plastically incompressible solids in a qualitative way (Toribio and Kharin, 2009). For this material, with $\alpha=0.28$, there is a decrease in the maximum absolute stress in comparison to 
(a)

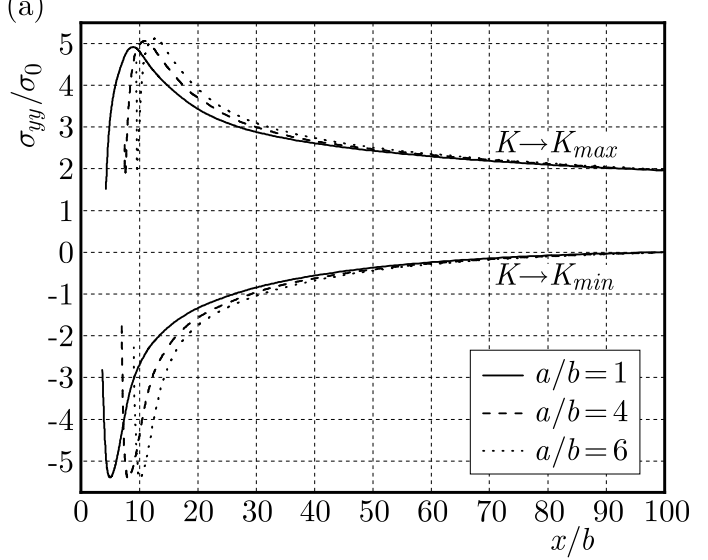

(b)

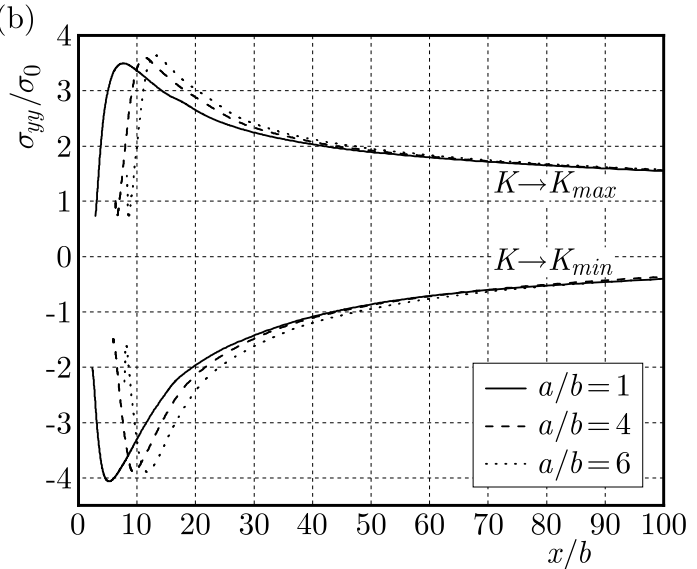

Fig. 8. Distribution of the near-tip normal stress $\sigma_{y y}$ for material B at high and low peak loads, 7th cycle: (a) plastically incompressible, (b) plastically compressible $(\alpha=0.28)$

(a)

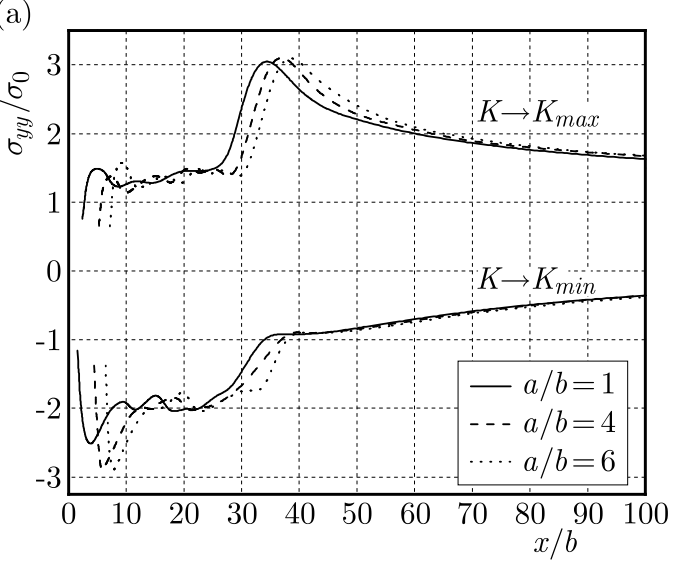

(b)

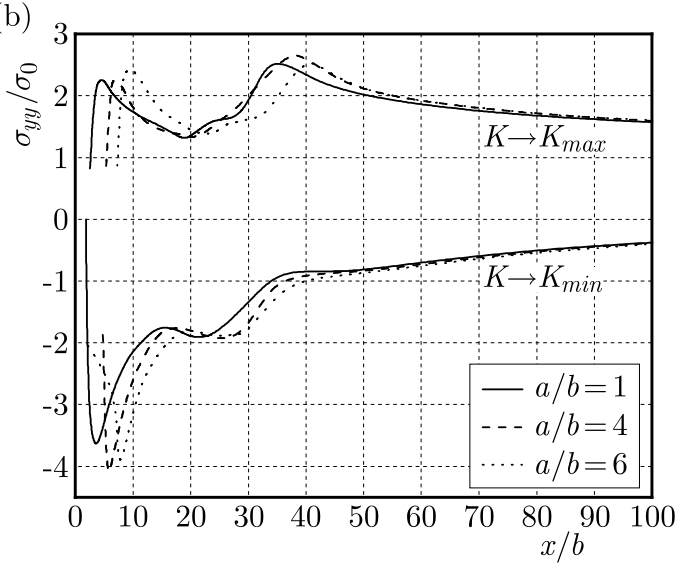

(c)

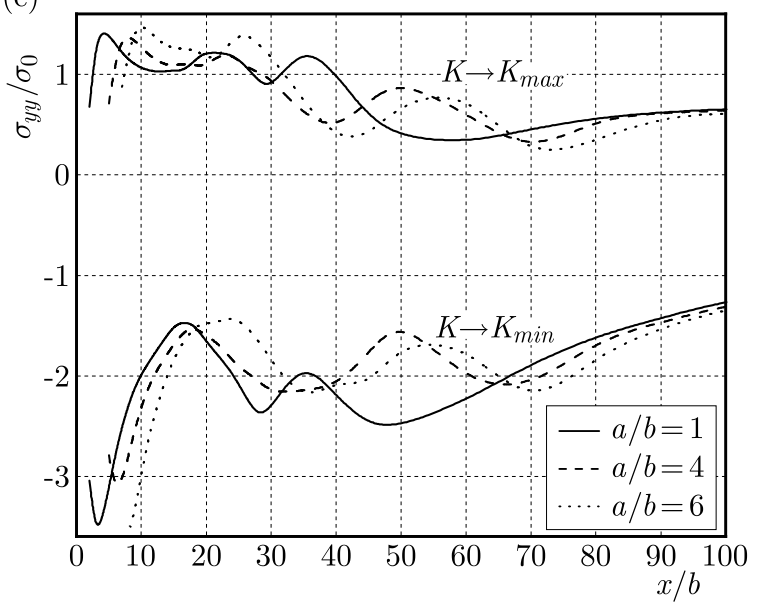

Fig. 9. Distribution of the near-tip normal stress $\sigma_{y y}$ for material E, plastically compressible $(\alpha=0.28)$ at high and low peak loads during (a) 1st cycle, (b) 7th cycle (without overload) and (c) 7 th cycle (with overload)

those of the plastically incompressible solid for both $K \rightarrow K_{\max }$ and $K \rightarrow K_{\min }$. The maximum absolute tensile and compressive stresses corresponding to $K \rightarrow K_{\max }$ and $K \rightarrow K_{\min }$ for the plastically incompressible solid are, respectively, $\left(\sigma_{y y}\right)_{\max }=5.0$ and $\left(\sigma_{y y}\right)_{\min }=5.5$, Fig. 8a. On the other hand, the corresponding stresses for the plastically compressible solid $(\alpha=0.28)$ are $\left(\sigma_{y y}\right)_{\max }=3.5$ and $\left(\sigma_{y y}\right)_{\min }=4$, Fig. 8b. The compressive stress is not reaching zero up to 
$x / b=100$ when $K \rightarrow K_{\text {min }}$ in Fig. 8b, however the same is nearly zero in Fig. 8a, and the cause of this may be the formation of enlarged size of a plastic zone for the plastically compressible solid. Because of plastic compressibility, some residual stress is present in the material after every load cycle.

Next, in material $\mathrm{E}$ with $\alpha=0.28$, where there is material softening followed by hardening, the near-tip normal stress $\sigma_{y y}$ distributions are not self-similar in nature for $K \rightarrow K_{\max }$ and $K \rightarrow K_{\min }$ in both the 1st and 7th load cycles, Fig. 9. Results were also generated for the plastically incompressible solid as well as other plastic compressibility levels. It has been detected that with a rise in the plastic compressibility level, self-similarity in the distribution pattern is destroyed more, and the peak $\sigma_{y y}$ value is reduced. Absolute tensile and compressive peak stress values are observed when the crack-tip curvature radius is increased from $a / b=1$ to $a / b=4$ and 6. Also, these absolute tensile and compressive extremes of stress in the 7 th cycle are greater when compared to the corresponding 1st cycle, Figs. 9a and 9b. Moreover, there are kinks in the stress distribution along the crack plane, and peaks in the normal stress distribution are observed a little away from the tip, and the cause of this possibly may be the intense plastic straining which emanates from the crack-tip (Khan et al., 2017). The crack-tip shapes govern the location of these kinks more or less. Here are the absolute values of $\left(\sigma_{y y}\right)_{\max }=3.25$ and $\left(\sigma_{y y}\right)_{\min }=2.75$ during the 1st cycle, Fig. 9a, and $\left(\sigma_{y y}\right)_{\max }=2.5$ and $\left(\sigma_{y y}\right)_{\min }=4.0$ during the 7 th cycle, Fig. 9b. The compressive stress is increased with the number of cycles due to the cumulative residual stress effect. After the application of the overload, the fluctuation in the stress distribution increases much. The peak values of the tensile and compressive stresses are reduced significantly in the 7th cycle, and the near-tip stress distribution with respect to the crack-tip curvature becomes more diverse from each other. After application of the overload, the amount of residual stress noticeably increases correspondingly to $K \rightarrow K_{\min }$.

\subsection{Distribution of crack growth stress $\left(\sigma_{x x}\right)$ along the symmetry plane}

Figure 10 describes the near-tip distribution of the crack growth normal stress $\sigma_{x x}$ along the $x$-axis at low and high peak loads during the 1st and 7th load cycle for material $\mathrm{E}$ with $\alpha=0.28$. Significant differences are observed in the distribution of $\sigma_{x x}$ when the crack-tip curvature radius is changed, but the initial crack-tip shape does not influence the far field stress. The absolute tensile and compressive extremes of stress corresponding to $K \rightarrow K_{\max }$ and $K \rightarrow K_{\min }$ significantly depend on the number of cycles. Absolute tensile and compressive $\sigma_{x x}$ stress values are maximum for each figure corresponding to the crack-tip shape with $a / b=6$. Kinks are again observed a little away from the crack-tip, and the cause of this may be the same intense plastic straining which emanates from the tip. The shape of the crack-tip more or less governs the location of these kinks. The absolute values of $\left(\sigma_{x x}\right)_{\max }=2.5$ and $\left(\sigma_{x x}\right)_{\min }=5.5$ for the 1st cycle and $\left(\sigma_{x x}\right)_{\max }=4.0$ and $\left(\sigma_{x x}\right)_{\min }=6.0$ for the 7 th cycle. After application of the overload, the tensile and compressive extreme values of the stresses increase significantly (much for the compressive stress). The fluctuation in the $\sigma_{x x}$ stress distribution increases along the crack plane and also with respect to the crack-tip shape. The amount of residual stress also markedly goes up corresponding to $K \rightarrow K_{\min }$.

\subsection{Distribution of hydrostatic stress $\left(\sigma_{h} / \sigma_{0}\right)$ along the symmetry plane}

Figure 11 shows the evolution of the near-tip hydrostatic stress distribution along the crack plane for material E. Very close to the crack-tip and inside the plastic zone, hydrostatic stress fields are similar to the elastic near-singular fields. Hydrostatic stress distribution in Fig. 11a is different from Fig. 11b. In Fig. 11a, changes in the crack-tip curvature radius make a change in the distribution of hydrostatic stress up to nearly $x / b=25$. On the other hand, for a plastically compressible solid, the crack curvature radius influences the mean stress distribution differently 
(a)

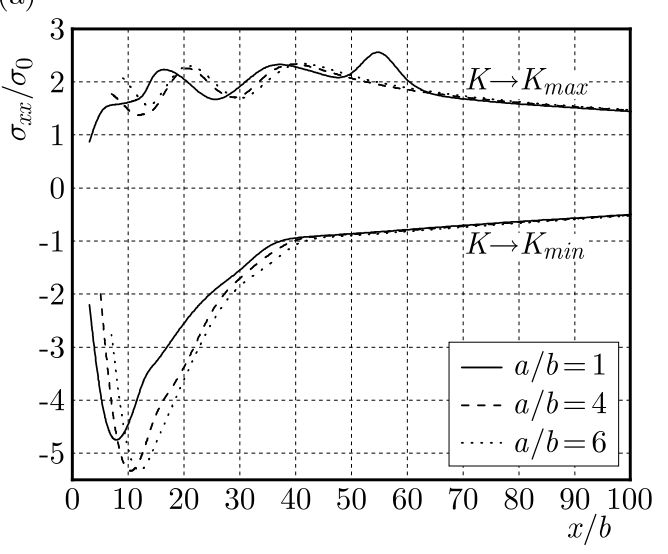

(b)

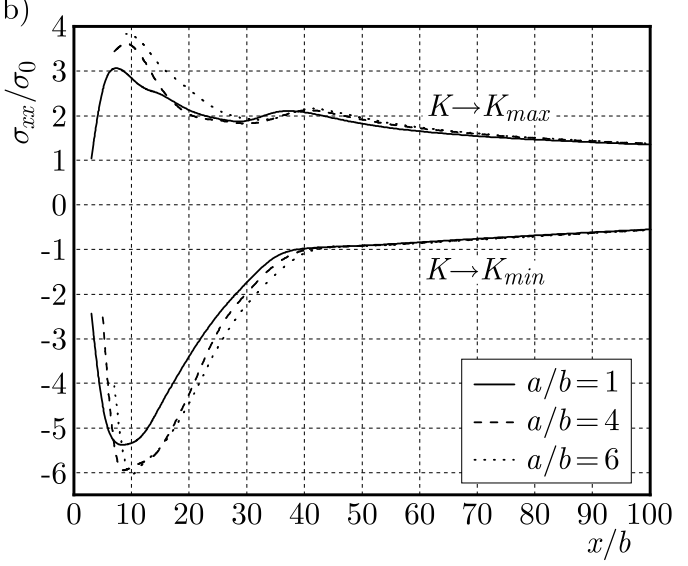

(c)

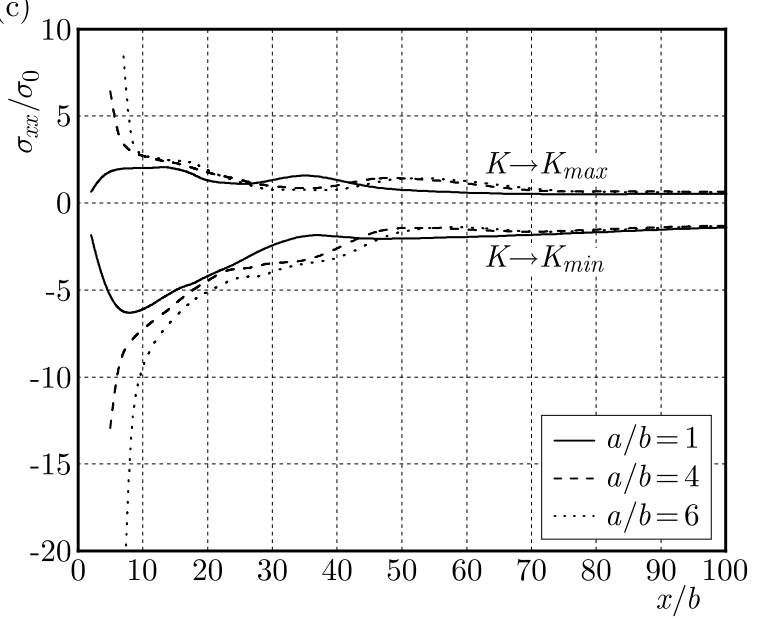

Fig. 10. Distribution of the near-tip normal stress $\sigma_{x x}$ for material E, plastically compressible solid ( $\alpha=0.28)$ at high and low peak loads (a) 1st cycle, (b) 7th cycle (without overload) and (c) 7th cycle (with overload)

up to nearly $x / b=40$, and correspondingly to $K \rightarrow K_{\min }$ the maximum value of the compressive stress is considerably higher for the tip with $a / b=6$ as compared to the same corresponding to $a / b=1$. Further, the overload increases both the tensile and compressive hydrostatic stresses to a great extent though the increase is greater for the compressive stress. The highest compressive stress is observed for the tip with $a / b=6$. Why the blunter notch leads to a higher crack growth rate may be explained from this observation also.

\section{Conclusions}

The present finite element deformation analysis of the near-tip fields of mode I crack with different initial crack-tip shapes for a plastically compressible hardening-softening-hardening solid subjected to fatigue loading with an overload permit us to list the following conclusions:

- Both the crack opening stress $\sigma_{y y}$ and crack growth stress $\sigma_{x x}$ corresponding to high and low peak loads in a cycle are sensitive to initial crack-tip shape, plastic compressibility as well as material or strain softening.

- Absolute tensile and compressive extremes of normal stresses, maximum hydrostatic and residual stresses occur for the maximum crack-tip curvature radius.

- As the compressive stresses generated correspondingly to $K \rightarrow K_{\min }$ are observed to be the highest for the crack-tip with the maximum curvature, the present $\sigma_{y y}$ and $\sigma_{x x}$ stress 
(a)

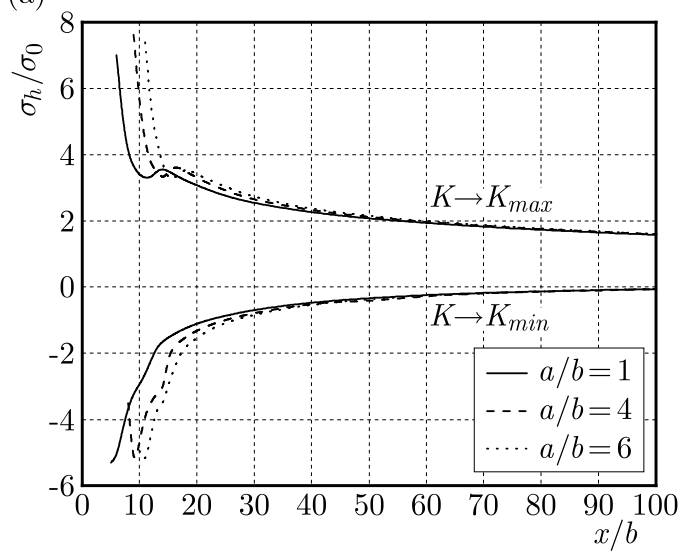

(b)

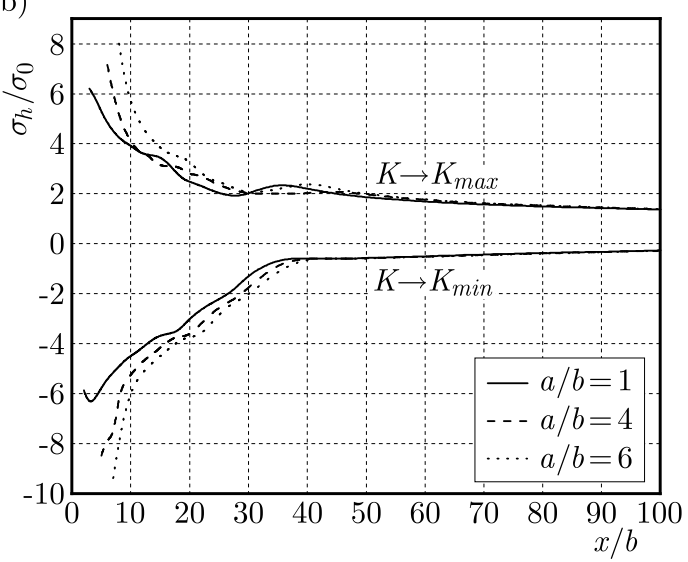

(c)

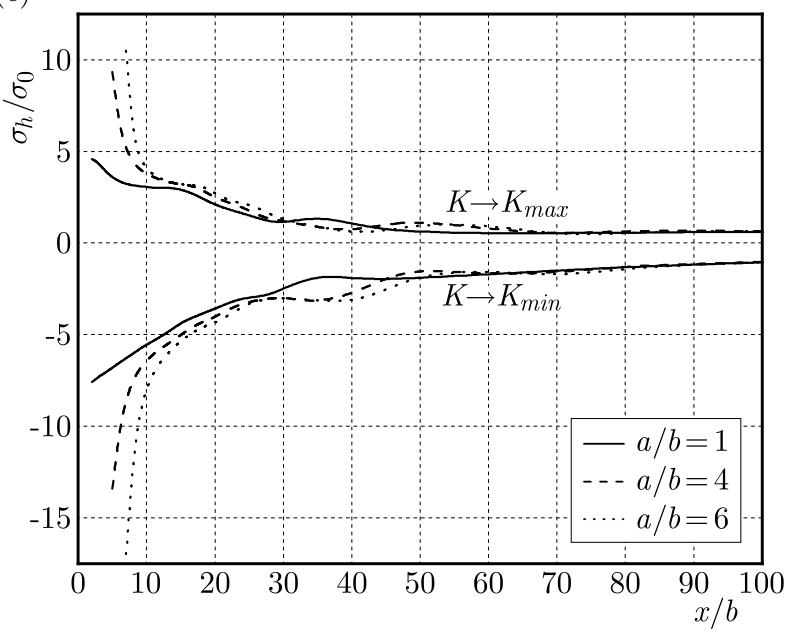

Fig. 11. Distribution of the near-tip hydrostatic stress in material E at high and low peak loads, 7th cycle (a) plastically incompressible, without overload, (b) plastically compressible $(\alpha=0.28)$, without overload, (c) plastically compressible $(\alpha=0.28)$, with overload

results as well as hydrostatic stress can explain why there is a bigger fatigue crack growth for the blunter notch as earlier observed by researchers.

- After application of the overload, the tensile and compressive extreme values of the $\sigma_{y y}$ stresses decrease, whereas $\sigma_{x x}$ stresses increase (especially compressive stress) considerably.

- Plastic compressibility leads to residual stress generation in the material after every load cycle.

- After application of the overload, the amount of residual stress noticeably increases correspondingly to $K \rightarrow K_{\min }$.

\section{References}

1. Alam M.I., Khan D., Mittal Y., Kumar S., 2019, Effect of crack tip shape on near-tip deformation and fields in plastically compressible solids, Journal of the Brazilian Society of Mechanical Sciences and Engineering, 41, 10, 441-1-15

2. Hutchens S.B., Needleman A., Greer J.R., 2011, Analysis of uniaxial compression of vertically aligned carbon nanotubes, Journal of the Mechanics and Physics of Solids, 59, 10, 2227-2237 
3. Khan D., Singh S., Needleman A., 2017, Finite deformation analysis of crack tip fields in plastically compressible hardening-softening-hardening solids, Acta Mechanica Sinica, 33, 1, $148-158$

4. Liu N., Drugan W.J., 1993, Finite deformation finite element analyses of tensile growing crack fields in elastic-plastic material, International Journal of Fracture, 61, 3, 189-210

5. Mohan N., Cheng J., Greer J.R., Needleman A., 2013, Uniaxial tension of a class of compressible solids with plastic non-normality, Journal of Applied Mechanics, 80, 4, 040912-1-8

6. Needleman A., Hutchens S.B., Mohan N., Greer J. R., 2012, Deformation of plastically compressible hardening-softening-hardening solids, Acta Mechanica Sinica, 28, 4, 1115-1124

7. Peirce D., Shih C.F., Needleman,, A., 1984, A tangent modulus method for rate dependent solids, Computers and Structures, 18, 5, 875-887

8. RicE J.R., 1968, A path independent integral and the approximate analysis of strain concentration by notches and cracks, Journal of Applied Mechanics, 35, 2, 379-386

9. Rozumek D., Macha E., Lazzarin P., Meneghetti G., 2006, Influence of notch (tip) radius on fatigue crack growth rate, Journal of Theoretical and Applied Mechanics, 44, 1, 127-137

10. Sadananda K., Vasudevan A.K., Holtz R.L., Lee E.U., 1999, Analysis of overload effects and related phenomena, International Journal of Fracture, 21, S233-46

11. Singh S., Khan D., 2018, On fatigue crack growth in plastically compressible hardening and hardening-softening-hardening solids using crack tip blunting, International Journal of Fracture, 213, 2, 139-155

12. Steuwer A., Rahman M., Shterenlikht A., Fitzpatrick M.E., Edwards L., Withers P.J., 2010, The evolution of crack-tip stresses during a fatigue overload event, Acta Materialia, 58, 4039-4052

13. Toribio T., Kharin V., 2009, Finite-deformation analysis of the crack-tip fields under cyclic loading, International Journal of Solids and Structures, 46, 9, 1937-1952 